\title{
The Corsican Mazzeru at the Dawn of XXI E Century: The End of a Shape of Shamanism?
}

\author{
Fogacci Tony \\ Professeur Université Anthropologie, Langues et Cultures Régionales, \\ Lari Vannina \\ MCF Anthropologie, Langues et Cultures Régionales, \\ UMR CNRS 6240 Laboratoire LISA - Université de Corse (France)
}

\author{
Doi:10.5901/ajis.2013.v2n8p764
}

\begin{abstract}
The mazzeru or the mazzera is a key character of the Corsican society, he is at the same time an intermediary psychopompe and an actor of the symbolic passage of the land of the living towards the world of the deaths. In Corsica, the said person mazzeru is a very real individual being a member of the community, which dreams mostly about a hunting during its sleep. During this dream, he brings (shoots) down an animal; at this precise moment his function of intermediary of the worlds, boatman of souls, is going to become a reality because when he turns the animal, the head represents a person who will soon die. This intermediary of the worlds is situated in the sociopolitical limits of the villages of the island, his powers him being similar in a genre of chamane. In Corsica, this phenomenon seems to have resisted partially the christianization and the numerous religious prohibitions, which were able neither to cover completely this archaic religion, nor to transform it. The mazzeru is situated after all in the center of a vision meadow and post-mortem specific, which makes perceive (collect) the death as a passage. We shall develop our reflection around the testimonies, the evolutions of this survival and the problem of its transmission at the time of the globalization.
\end{abstract}

Il est admis que la prise en compte de la mort remonte à la préhistoire : dès lors que l'homme prend soin de la dépouille et ritualise, par un enterrement la gestion du corps, l'acte va s'accompagner également de la gestion de l'au de là, de la prise de conscience d'une possibilité d'une vie autre. Ainsi on va réserver aux morts des espaces particuliers, la plupart du temps proche de l'espace des vivants mais bel et bien délimités; des temps différents vont également être perçus comme des jonctions entre un monde et l'autre, lesquels semblent entrelacés et non séparés. On sait aujourd'hui qu'en plus d'un certain nombre de rituels liés à la préparation du corps (ocre rouge) comme à la mise en terre (position, choix du lieu, présence de pollens supposant l'ajout de fleur avant la fermeture de la tombe ou la mise en terre), des outils, des ornements, des "armes », et même des mandibules et des ossements d'animaux accompagnaient le défunt vers son autre monde.

En Corse, une croyance concernant la composition de l'être humain met en évidence cette gestion du passage de la vie à mort, d'un espace à un autre, d'un monde à l'autre. En effet, on considère que l'être humain est composé de trois « corps » : u corpu à sali, le corps physique, ou l'enveloppe carnée, u corpu à spiritu, l'esprit, et l'anima, l'âme ; le corps de chair, tangible, est celui qui nous représente dans le monde des vivants, l'esprit est notre représentation dans le monde des rêves mais également lors du passage ultime dans l'autre monde, sorte de véhicule permettant à l'âme le franchissement vers l'Altru Latu, l'autre côté, l'Autre Monde, l'âme étant notre représentation dans l'au-delà. Ce rôle de passeur est endossé par un être désigné par le terme mazzeru, qui deviendra générique au fil du temps ; il désigne donc un personnage au rôle psychopompe, c'est à dire qui a le devoir (ou le don ?) de guider l'esprit vers le monde des morts, de permettre ce franchissement vers l'autre côté. Nous considérons par cet acte de séparation que le corps, ainsi privé de son âme ne peut plus vivre, le passage définitif provoque alors la mort du corps physique. En s'éveillant, le mazzeru retrouve son esprit, il peut ainsi se remémorer son rêve et comprendre ainsi qui va mourir. Si on peut considérer de manière simpliste que le mazzeru possède le don de voir la mort en rêve, il convient de souligner que son rôle consiste plus précisément à détacher la vie du vivant, de conduire l'âme du mort dans l'Autre Monde.

Le mot mazzeru découle sans doute de mazza, massue ou maillet, outil de bois qui rappelle l'ancienne pratique consistant à assommer le moribond afin de l'aider à franchir lorsque l'âme passée, le corps tarde à s'éteindre. Ammazzà, tuer reprend également cette idée d'assommoir. 
Plusieurs termes employés pour désigner cet initié lié à la mort nous amènent aussi vers l'idée de guerrier : $u$ lancieru, le lancier, tout comme les termes de culpadori et acciaccadori participent également à la réputation de tueur qu'endosse u mazzeru.

Ainsi, de nombreux de rêves symboliques rapportent des scènes de chasse où le mazzeru, retournant le corps de l'animal qu'il vient d'abattre, reconnaît la personne qui va mourir. L'aspect violent, sanglant prend aussi une forme plus conviviale par le biais d'un autre type de rêve mettant en en scène également une pratique culturelle réelle, celle du repas de deuil. A manghjaria désigne ainsi le repas où se retrouvent la famille et les alliés du défunt; on tuait, à cette occasion, un veau ou un cochon selon la région ou la saison. Le pendant révée est désigné par le même terme et met en scène une tablée où se retrouvent en esprits les mazzeri . ils y consomment également de la viande, parfois crue ou bien des racines. II s'agit ici de la représentation du corps du défunt à venir ainsi partagé et ingéré par la communauté. L'Eglise a d'ailleurs tenté d'interdire le repas de deuil justement à cause de cet aspect symbolique ${ }^{1}$.Cependant, s'il s'agit des types de rêves les plus fréquents, la forme que peuvent revêtir ces " prémonitions », restent intimement liées à la personne qui rêve, à celle dont elle rêvé, chacune s'appuie sur un système symbolique propre, parfois fort difficile à expliquer. Le mort peut être une personne qui franchit un cours d'eau ou bien qui gravit une colline, ce peut être une flèche tirée d'on ne sait où, ou encore la pierre que l'on jette au fond d'un pozzu, d'un trou d'eau, ou bien encore l'animal qui s'enfuit et disparaît, plusieurs fois de suite et toujours en un espace symbolique ou ayant une importance pour la personne qui doit mourir ; c'est au réveil, à partir de ces indices, de ces messages qu'u mazzeru va justement comprendre qui va mourir. Celui qui agit et qui a accès à ce monde sait interpréter et faire la différence entre un rêve ordinaire et un rêve que l'on qualifie de manière inappropriée de " prémonitoire ». En effet le terme de prémonition ne peut résumer le sens des rêves des mazzeri. II signifie avertissement, pressentiment et intuition, il a le sens de deviner l'avenir, de voir avant. Ce sens est en fait incomplet car il ne permet pas d'exprimer qu'il y a, au delà de la vision rêvée d'un futur, un acte qui initie ce futur en même temps qu'il l'indique à celui qui rêve et donc qui le commet. Le mazzeru agit sur l'esprit, puis l'âme, ce qui a des conséquences sur le corps physique de la personne représentée en rêve. Le fait de comprendre un rêve est bien une prémonition, mais ce terme est la finalité de tout un processus actif qui renvoie à l'inconscient et n'englobe donc pas la réalité rêvée, et la symbolique qui en découle.

Le mazzeru prend aussi une dimension plus grande encore dès lors qu'il endosse le rôle de meneur. II dirige a Cumpagnia di i morti ou a Squadra d'Arozza, qui sont les processions venues pour chercher l'esprit de celui qui doit mourir afin de le conduire aux portes du monde des morts². II s'agit ici d'une parodie d'enterrement, et il faut arrêter la procession aux ports de l'église afin de sauver la personne concernée. Il est également conseillé de ne pas se saisir d'un éventuel objet tendu par l'un des confrères (un cierge la plupart du temps) car cet acte enclenche alors le début d'une initiation, l'homme se retrouve « condamné » à sortir en esprit, participant aux processions, allant chercher les siens afin des les guider jusqu'aux frontières de l'au delà. Celui qui dirige cette confrérie composée de morts, d'esprits et d'animi in pena, l'âme des morts sans sépulture est désignée par le terme de Capistrigonu. Le terme strionu ou strigonu, dérive de streia, stria ou strega dont nous allons définir le rôle plus loin. Il signifie « sorcier », cette entité étant aussi désignée de différentes manières et renvoie également aux différentes dénominations du mazzeru, tout comme magu et ses dérivés - maga, magonu, magaccia ${ }^{3}$.

Le regroupement de différentes forces, de différentes entités se retrouve également dans les mandraghi, les batailles nocturnes. II s'agit des batailles opposant les mazzeri des villages rivaux et qui ont lieu dans la nuit du trente et un juillet au premier août, I mazzeri combattent les uns contre les autres, souvent à l'aide du tarabuchju, l'asphodèle, et l'issue du combat déterminera le village qui perdra le moins d'âmes dans l'année et la qualité comme la quantité des récoltes. On remarque que ce combat symbolique dans le monde des rêves a des effets dans la réalité ainsi qu'une alliance Vie/Mort concrétisée par l'enjeu des moissons et de la «fin » humaine, et qui illustre bien l'ambivalence du mazzeru. De ce fait, n'est pas seulement lié à la Mort, mais également à la Vie, indissociables moments formant le Destin.

\footnotetext{
${ }^{1}$ Cancellieri J.A. Bulletin de la société des sciences historiques et naturelles de la Corse $n^{\circ} 652,1985$

${ }^{2}$ Ces processions s'apparentent à la Compagnie Hennequin, ou Hellequin de la tradition française représentant le cortège des morts mené par un personnage à figure humaine, vêtu de peaux de bouc, de chèvre ou de loup.

${ }^{3}$ Ce terme renvoie aussi au masque, rejoignant la notion de double, puisque le masque montre, d'une certaine manière l'autre visage de l'humain, la révélation de son inconscient.
} 
Pour résumer le rôle du mazzeru, on soulignera le fait qu'il n'est pas de tuer, mais d'aider au passage, au franchissement, car c'est de ce moment que dépendra la vie dans l'au-delà. La religion chrétienne nous dit que c'est la vie sur Terre qui détermine la place de l'Homme dans l'autre monde, elle met ainsi en place de nombreux devoirs et interdits qui, s'ils sont respectés assurent un au-delà paradisiaque. Les religiosités pré-chrétiennes expliquent que c'est aussi le passage qui détermine le repos de l'âme, même si la vie humaine l'influence aussi. La punition, c'est d'errer sur Terre, entre deux mondes, sans attache, et c'est ce qui se passe si le mazzeru fait mal son travail, si on le dérange dans son sommeil actif.

Autre personnage psychopompe, la Strega, la sorcière, est donc également un être humain qui a la capacité de se dédoubler ; de la même manière que les mazzeri, elle a un sommeil actif, pendant lequel son esprit quitte son corps pour se rendre aux frontières entre les mondes. Elle fait partie elle aussi de la compagnie des morts-, que mène $u$ capistrigonu. II s'agit donc d'un être intermédiaire qui se situe aux endroits de franchissements comme les ponts, les cols, les cours d'eau, les pozzi ; mais contrairement au mazzeru qui semble subir le don qu'il possède sans pratiquement aucun moyen de le maîtriser, il est dit que la strega semble capable de maîtriser ses agissements nocturnes lorsqu'elle sort en esprit.

C'est un personnage semblable à d'autres intermédiaires perçu de par ce fait par la communauté de manière plus négative. On l'accuse d'ailleurs de boire le sang des nouveau-nés, cette pratique vampire lui servant à se régénérer. A contrario, elle est aussi guérisseuse. Son rôle est donc très ambiguë, car si elle voit la mort, si même elle la provoque, et qu'elle attaque volontairement sa proie comme un animal, elle est aussi capable de soigner, et est sollicitée au besoin. Elle provoque aussi bien effroi que fascination et reste moins bien intégrée dans la société que le mazzeru même si pour tous les deux, le don de voyager entre les mondes est mal perçu. Nous l'avons vu, l'Eglise a contribué à créer la croyance que le fait d'avoir cette capacité de circuler découle d'un baptême non valide. En effet, des paroles mal prononcées, un oubli du curé peuvent faire que le nouveau-né n'entre pas entièrement dans la société des hommes, puisque tel est le but du baptême. Ainsi il erre entre deux mondes et « subit », du moins pour l'Eglise, sa capacité à franchir les limites entre les mondes. Le don de « voir » est compris comme un maléfice, véritable manifestation du Malin que certaines prières peuvent atténuer, du moins pour un temps. Or on soupçonne la strega de tuer volontairement, « consciemment », de jeter des sorts.

I mazzeri et $i$ sdreii de Corse, les strugei de Slovénie, les Molphars des Carpates, les chamans sibériens, africains ou amazoniens, les hommes-médecine d'Amérique du nord, i benandanti du Friul, i lupi mannari, les loups-garous, l'erchiti de Sardaigne, quels que soient les noms qu'on leur donne, tous appartiennent à la même catégorie universelle, celle des guérisseurs et de ceux qui voient au delà du visible. Ce qui peut aujourd'hui disparaître, c'est simplement le savoir qui accompagne ces actes nocturnes. Effacer certaines croyances, en détourner d'autres, les diaboliser, permet à l'Eglise de confisquer les outils qui permettent d'interpréter les rêves symboliques mais pas d'empêcher que ces rêves aient lieu, ceci afin d'imposer ses propres règles et d'être l'unique intermédiaire entre en bas et en haut.

En 1975 déjà, le chercheur Roccu MULTEDO, à l'origine d'études sur les croyances magico-religieuses en Corse, établit un premier parallèle entre le « mazzeru » et le chaman (in « Le 'mazzérisme' et le folklore magique de la Corse, ADECEC, Cervione, 1975, page 44). II reprend la comparaison dans "Le mazzérisme, un chamanisme corse » édité par l'Accademia Corsa, Nice, 1981, deuxième édition : Bastia, 1982, puis dans le fascicule "Le mazzérisme est-il un chamanisme corse ? » (C.R.E.T, 1986, 2 ème édition août 1987, Bretagne)

Comment peut-on définir le « mazzeru » ou la « mazzera » ? Personnage clé de la société corse, il est à la fois un intermédiaire et un acteur du passage symbolique du monde des vivants vers le monde des morts. En Corse, la personne qui est dite «mazzeru » ou «mazzera » est un individu bien réel, homme ou femme, faisant partie de la communauté, qui rêve le plus souvent d'une chasse pendant son sommeil, de là l'analogie faite avec le chaman, qui lui, peut cumuler plusieurs fonctions : prêtre, sorcier, magicien, devin, et guérisseur traditionnel.

Lors de ce rêve, le «mazzeru » chasse ou participe à une battue collective, abat un animal, le plus souvent un sanglier, et il est vrai qu'en Corse la battue au sanglier est remarquable dans son interaction profonde avec l'imaginaire collectif. Au poste de sanglier, le chasseur doit faire face à l'imprévisible, c'est un face à face avec la mort qu'il va donner, en ce sens le parallèle avec la chasse du mazzeru, personne de la communauté qui voit l'avenir dans des rêves, est logique. Certains ressentent la rencontre avec l'animal comme une rencontre avec la mort, le destin. Dans la pratique, chaque chasseur est posté à un endroit de passage, a posta, lieu de franchissement où aboutissent l'andati, les sentiers tracés par les sangliers, endroits où se rejoignent le maximum de voies propres aux animaux, lieu du choix et de la rencontre avec le destin.

Certains témoignages mentionnent également d'autres animaux : chien, lapin, cochon, etc...C'est à ce moment précis que la fonction d'intermédiaire entre les mondes, de passeur d'âmes va se concrétiser car lorsque le « mazzeru » 
retourne l'animal, sa tête représente un proche de la famille, ou du village, une personne qui est « la victime » choisie par le destin, lors du rêve de cette chasse nocturne. Mais le mazzeru a également une fonction salvatrice et peut ainsi sauver la future victime en l'empêchant de franchir l'entrée d'une église ou en faisant retourner le cercueil d'un enterrement fantôme en s'asseyant devant la porte de sa maison.

Le « mazzérisme » est un mot créé par Roccu Multedo à partir du mot corse « mazzeru », ainsi le phénomène à proprement dit des mazzeri peut-être donc apparenté à une certaine forme de chamanisme, tout en sachant que le chamanisme connait de nombreuses variantes sur la surface de la terre. Ce phénomène du 'mazzérisme' en Corse semble avoir résisté en partie à la christianisation, aux nombreux interdits religieux, qui n'ont pu ni recouvrir complètement cette religion archaïque, ni la transformer.

En vérité, nous ne sommes pas ici in présence d'un sorcier chaman traditionnel revêtu d'une peau d'animal ou d'un vêtement comportant des symboles représentant un animal. Mais la relation homme-animal y est bien présente puisque le mazzeru peut prendre la forme d'un animal lors d'une rencontre avec l'humain, jouant alors son rôle d'accompagnateur dans l'autre monde.

Tel le chaman il s'affirme comme un intermédiaire entre les hommes et les esprits, et sa relation avec l'animal et la nature est particulière. II voit un animal mort lors de ses visions mais peut très bien se transformer en animal (chat, chien, renard) lors de sa rencontre au seuil des mondes avec la victime.

Comment devient-on mazzeru ? Plusieurs hypothèses sont évoquées. On mentionne tout d'abord une erreur de prononciation des prières de la part du parrain ou de la marraine pendant le baptême de celui qui deviendra effectivement mazzeru, ou alors un don inné que l'on ne peut esquiver, car le mazzeru ne peut aller contre le choix du destin. De même au Frioul, ceux qui naissaient coiffés c'est-à-dire recouverts de la membrane amniotique, ne pouvaient échapper à leur fonction de benandante, celle de voyager en esprit pour lutter contre les mauvais sorciers, les malandanti. Le mazzeru n'est pas responsable de la mort de la victime qu'il aperçoit lors de sa chasse, il assume cependant sa fonction de régulateur de la mort, car il l'accompagne.

Certains témoignages émettent l'hypothèse d'une véritable initiation comme dans les sociétés secrètes, mais on dispose de peu d'éléments à l'heure actuelle pour étayer cette version. Enfin on évoque une affiliation par le rêve, ce qui le rapproche un peu plus de la définition commune du chaman. Mais ce sont des personnes ayant toutes les prédispositions innées à exercer cette fonction, ce pouvoir psychopompe.

Ainsi à travers des lieux propices au franchissement, à la métamorphose, finalement à la périphérie du changement, apparaissent ces êtres de la limite, des personnes détenant un pouvoir, exerçant une fonction, activant ce 'franchissement' par une métamorphose en chat ou en chien.

Les mazzeri (hommes et femmes) se déplaçant souvent en société, sont censés être accompagnés de leur meute de chiens, toujours dans le but de chasser. Des témoignages évoquent précisément toutes les modalités d'une battue au sanglier, son organisation nocturne en rêve est la réplique d'une vrai battue avec des mazzeri chargés de rabattre le gibier, d'autres faisant les voix, et les mazzeri tireurs qui tuent l'animal lors du franchissement du poste qui bien évidemment est dans ce cas un passage des mondes tout à fait symbolique.

Cet intermédiaire des mondes va se situer aux lieux de franchissement, aux crêtes et aux limites sociopolitiques des villages ou dans les fleuves. Toutes ces limites sont des crêtes de montagnes, des cours d'eau, des gués, des ponts, et donc considérés comme des espaces de transition et de conflits entre les mondes.

Le mazzeru se situe en définitive au centre d'une vision pré et post mortem spécifique, qui fait percevoir la mort comme un passage, sans doute les traces d'une religion archaïque bien plus importante, avec tout un complexe de croyances associées : feux rituels, purifications, batailles symboliques annuelles, culte de l'ancestralité.

Sa fonction de véritable régulateur de la mort apparait de manière significative à travers les croyances de la «Calendula d'aostu » ou « Mandraca » de la nuit du 31 juillet qui voit s'opposer lors de luttes imaginaires, les mazzeri de villages et régions différentes. Cette croyance transmise par de nombreuses générations, mentionne une lutte annuelle collective de sociétés de mazzeri.

L'enjeu est de taille: il s'agit d'une bataille fictive en rêve où les mazzeri sont les représentants de leur communauté qui vont lutter pour la survie des lignées de leur groupe. Les affrontements ont pour cadre les espaces délimitant les régions ou villages, en général les cols de franchissement des vallées proches. Ces seuils sont bien évidemment des entrées symboliques faisant communiquer le monde des vivants avec l'au-delà du monde des morts.

Lors de ces batailles annuelles qui se déroulent dans la Corse entière, la mortalité sera plus forte dans le camp des perdants. D'après la tradition orale, c'est la direction prise par le brouillard matinal qui annonçait la victoire d'un camp ou de l'autre. Ainsi, le signal indiquant le camp victorieux est constitué par un signe naturel, le sens de la fumée qui descend du col principal de la vallée vers un camp ou vers l'autre. 
La date n'est pas anodine, les batailles ont lieu dans la nuit du 31 juillet au 1er août, époque la plus forte de la canicule d'été, période propice à toute perte de limite depuis les temps les plus reculés. Cette date correspond à la conjonction symbolique des mondes des vivants et des morts, un espace flou, en rupture, véritable moment charnière de l'année similaire au $1^{\text {er }}$ novembre, période de la fête des morts.

Agissant au nom de la communauté villageoise ou de la vallée qu'elles représentent, les sociétés de mazzeri sont engagées dans des batailles fictives sur les points culminants des montagnes. Peut-être que la bataille est analogiquement comparable à l'initiation des bouriates de Sibérie constituée par l'ascension d'un arbre qui est symboliquement l'axe du monde, acte rituel indiquant l'entrée du chaman dans sa fonction, et donc une ascension mythique le long de l'axe du monde.

Ces batailles connaissent une grande variété linguistique au niveau de leur appellation : a mandraca, a battaglia di i mazzeri, a calendula, parfois a battaglia di i morti. II faut dire que de nombreux toponymes cadastrés et connus de la tradition orale évoquent les lieux de ces batailles et confirment l'existence de la croyance : A Bocca à a Battaglia (le Col de la bataille), A Bocca à a Catarazza (le col de la trappe, lieu de passage symbolique) ainsi que beaucoup d'autres. On remarquera d'ailleurs que plusieurs de ces cols ont sans doute été christianisés et portent fréquemment le vocable de Saint Pierre.

Le lieu et la date sont fondamentaux, les batailles fictives se situent sur les sommets des montagnes séparant les communautés, aux limites sociopolitiques des villages et vallées. Car la rencontre avec le mazzeru a lieu en général dans des lieux de franchissement : ponts, gués, fleuves, entrées d'églises, crêtes, il est un passeur symbolique entre les mondes.

Lors de ces batailles nocturnes, les mazzeri s'affrontent avec des tiges d'asphodèles, ou même d'autres plantes, comme l'osier, selon la variété des témoignages dont on dispose dans les microrégions de l'île. Dans le village d'U Salice c'est l'immortelle qui est utilisée le même soir mais cette fois par les villageois dans un but de purification et de protection contre les mazzeri. On mentionne également des tibias, des os ou des bouts de bois utilisés lors de ces batailles cycliques.

L'asphodèle qui revient le plus souvent est évidemment lié symboliquement à la mort, il était planté près des tombes en Grèce, la Plaine des asphodèles était le lieu où la majorité des morts y demeuraient pour l'éternité.

Pendant la nuit de la bataille du 31 juillet, il y a un rituel du feu qui est une pratique à la fois de purification et de protection. Ce feu dénommé $U$ focu di i mazzeri est allumé devant les portes ou sur la place de l'église dans les villages, soit pour que les mazzeri puissent voir leur chemin, soit pour les expulser de la communauté ennemie, ou bien encore pour que les mazzeri vaincus puissent venir se régénérer à ce feu collectif. C'est qu'ici tout est ambivalence et le feu est un rite qui renforce la perméabilité entre les deux mondes, celui des morts et celui des vivants.

Le mazzeru est un personnage central de la tradition corse, dont certaines fonctions ont peut-être disparu, il est au centre d'un ensemble culturel plus complexe dont on ne saisit plus l'importance aujourd'hui. La polyvalence d'attributions et les pouvoirs du mazzeru l'apparentent à un genre de chaman : il peut prédire, chasser dans ses rêves, se transformer en animal, guérir et l'on notera la permanence de son pouvoir psychopompe. Son côté passif lors des rêves de chasse ne peut occulter cette fonction active qui le voit intervenir comme le vrai régulateur de la mortalité annuelle des communautés, le principal acteur de cet archétype cyclique, le témoin essentiel d'une vision de l'au-delà qui a perduré à travers les siècles.

\section{Bibliographie Indicative}

Atzori, Mario. Tradizioni popolari della Sardegna. EDES, 1997

Bachelard, Gaston. La Poétique de l'espace. Paris : PUF, 1957

Bachelard, Gaston. La Poétique de la rêverie. Paris : PUF, 1960

Balandier, Georges. Le dédale, pour en finir avec le XXème siècle. Paris, Fayard 1994

Botero, Jean. L'épopée de Gilgamesh. Paris, Gallimard, 1992

Braudel, Fernand. La Méditerranée et le monde Méditerranéen. Paris : Armand Colin, 1 ère éd. 1949, 1982, T.1 et T.2

Casanova, Jean Baptiste. Histoire de l'Eglise de Corse, Ajaccio, 1931-1939

De Martino, E. Italie du sud et magie. Paris : Gallimard, 1963.

Ginsburg, Carlo. Les batailles nocturnes. Paris, Flammarion, réed.1984

Ginsburg, Carlo. Le sabbat des sorcières. Paris, NRF Gallimard, 1992

Hall, Edward. T. La dimension cachée. Paris : Seuil 1971, Réed. 1978

Lari, Vannina. Parà a Sorti. Objets et rituels de protection de Corse. Dumane. 2007

Multedo, Roccu. Le nouveau folklore magique de la Corse. Stamperia Sammarcelli 1998

Lecouteux, Claude. Chasses fantastiques et cohortes de la nuit au Moyen Age. Imago, 1999. 
Leroi-Gourhan, André. Les religions de la préhistoire. Paris : PUF. 1995

Levi-Strauss, Claude. Des symboles et leurs doubles. Paris, Plon, 1989

Morin, Edgar. L'homme et la mort. [1951], Paris, Seuil, 1970

Philibert, Myriam. Mort et immortalité, de la préhistoire au Moyen Age. Editions du Rocher, 2002

\section{Articles}

Cancellieri J.A. Bulletin de la société des sciences historiques et naturelles de la corse $n^{\circ} 652,1985$

Caisson, Max. « La mort en Corse et dans les sociétés méditerranéennes » in Etudes corses, n 12/13, 1979.

Fogacci, Tony. « chasse » in Encyclopaediae Corsicae vol. Anthropologie, Bastia : Dumane 2004.

Fogacci, Tony. « Mort » in Encyclopaediae Corsicae vol. Anthropologie, Bastia : Dumane 2004.

Lari, Vannina. « Mazzeri » in Encyclopaediae Corsicae vol. Anthropologie, Bastia : Dumane 2004.

Lari, Vannina. « Strega » in Encyclopaediae Corsicae vol. Anthropologie, Bastia : Dumane 2004.

Moracchini-Mazel, Geneviève. « les saints patrons honorés en Corse et la christianisation de cultes antérieurs » Etudes corses $n^{\circ} 62$, 2006

Santini, Don-Mathieu. « La narration traditionnelle » in Encyclopaediae Corsicae vol. Anthropologie, Bastia : Dumane 2004.

Salini, Dominique. « Religion » in Encyclopaediae Corsicae, vol. Anthropologie, Bastia: Dumane, 2004.

\section{Thèses}

Fogacci, Tony. U ritu di a morti in Corsica. Thèse de Doctorat, Université de Corse, 1993.

Lantieri, Françoise. Les procès de sorcellerie en Corse. Thèse de Doctorat, Université de Corse, 1989.

Lari, Vannina. I fiuri di u puteri in l'imaghiinariu isulanu : morti, sorti è tramutà. Thèse de Doctorat, Université de Corse, 2007.

Santini, Don-Mathieu A fola corsa trà identità è universalità. Thèse de doctorat, Université de Corse, 1998. 